Results Among 323 participants, 89\% were female, 39\% Asian, 11\% African American, 22\% Hispanic of any race, and $29 \%$ White. Mean age was $45 \pm 14$; mean age at diagnosis 29 \pm 12 . Nearly half of respondents had a college degree. SDI at the baseline study visit ranged from 0 to 10 points, mean 1.8 $\pm 2.0 ; 70 \%$ of the cohort had $\mathrm{SDI}>0$. The regression model showed strong evidence $(p=0.01)$ for interaction of age of diagnosis with race/ethnicity. As seen in the figure 1, SDI scores in racial/ethnic minorities were much higher among those diagnosed at younger ages; this relationship was not seen among whites.

Conclusions In this multi-ethnic cohort of SLE patients, the association of diagnosis age and disease damage varied according to race/ethnicity, with whites diagnosed at younger ages accumulating less damage than those in other racial/ethnic groups diagnosed at comparable ages. Future research should examine if these differences are due to phenotypic differences among the groups, diagnostic delays, or other access to care issues.

Funding Source(s): Centers for Disease Control and Prevention (U01 DP005120)

\section{RELATIONSHIP BETWEEN SERUM LEVEL OF RENALASE AND LUPUS NEPHRITIS ACTIVITY}

Yasser Abdelmotaleb*, Khaled Zaky, Khaled Nofal. Al Azhar University, Egypt

\subsection{6/lupus-2019-Ism.214}

Background (LN)Lupus nephritis is a major risk for overall morbidity and mortality in SLE (Systemic lupus erythromatosis), and despite potent anti-inflammatory and immunosuppressive therapies still ends in Chronic Kidney Disease(CKD) or End Stage Renal Disease (ESRD) for too many patients. Renalase is a novel, kidney secreted cytokine-like protein that promotes cell survival.

Aim of the work studying the relationship between level of Human Serum Renalase (RNLS) with $\mathrm{LN}$ and its role in the disease activity and progression.

Methods For The current cross-sectional study 23 healthy controls and 48 patients with LN were screened and 30 subjects were selected These patients were subdivided into two equal groups according to disease activity measured by SLEDAI (SLE Disease Activity Index). Human Serum Renalase (RNLS) concentration was measured by a highly sensitive, commercial sandwich enzyme immunoassay which uses (RNLS) antibody to capture Renalase from serum. Assessment before and after treatment was done for 17 patients who received prednisone and immunosuppressive therapy were recruited and followed up for three months to evaluate the serum renalase levels before and after treatment.

Results The level of renalase was significantly higher in LN patients compared to healthy controls, ( $\mathrm{P}$ value $<0.001)$. Moreover, patients with active $\mathrm{LN}$ had higher serum renalase levels compared to patients with inactive $\mathrm{LN}(\mathrm{P}$ value $<0.005)$ Serum renalase levels were positively correlated with SLEDAI, 24 hour urine protein excretion, ds-DNA and ESR and CRP but inversely correlated with serum C3 and the class (especially in prolferative type (Class III, IV, more than class V). Renalase amounts decreased significantly after three-months of standard therapy. Also we found there is insignificant difference of renlase level according to treatment by MMF(mycophenolate mofetil) and Cyclophosphamide during and after activity ( $\mathrm{P}$ value $=0.655,0.550)$

Conclusions Serum renalase levels were correlated with disease activity in LN. Serum renalase might serve as a potential indicator for disease activity in LN.

\section{FIRST-IN-MAN STUDY EVALUATING THE SAFETY, TOLERABILITY, PHARMACOKINETICS AND CONCENTRATIONQT ANALYSIS OF THE NOVEL BTK INHIBITOR EVOBRUTINIB (M2951) IN HEALTHY VOLUNTEERS}

${ }^{1}$ Andreas D Becker, ${ }^{2}$ Emily C Martin, ${ }^{3}$ Victor Ona, ${ }^{4}$ David Y Mitchell, ${ }^{2}$ Anand C Patel ${ }^{*}$ ${ }^{5}$ Andreas Johne. ${ }^{1}$ Merck KGaA, Darmstadt, Germany; ${ }^{2} E M D$ Serono Research and Development Institute, Inc., Billerica, MA, USA; ${ }^{3} E M D$ Serono Research and Development Institute, Inc. (a business of Merck KGaA, Darmstadt, Germany), Billerica, MA, USA; ${ }^{4}$ Nuventra Pharma Sciences, Broomfield, CO, USA; ${ }^{5}$ Global Clinical Development, Merck KGaA, Darmstadt, Germany

\subsection{6/lupus-2019-|sm.215}

Background Brutons tyrosine kinase (BTK) regulates B-cell receptor signaling and is a rational target for B-cell driven pathologies, including SLE. Evobrutinib (M2951) is a highly specific, oral inhibitor of BTK that demonstrated efficacy in preclinical models of autoimmune disease. This first-in-man study investigated the safety, tolerability, and pharmacokinetics (PK) of evobrutinib in healthy volunteers and examined the relationship between evobrutinib exposure and changes in QT interval.

Methods This was a single-center, Phase I, double-blind, placebo-controlled trial. In Part 1, 48 healthy participants in six successive dose cohorts $(25,50,100,200,350,500 \mathrm{mg})$ were randomized $(6: 2)$ to a single oral dose of evobrutinib or placebo. In Part 2, 36 participants in three ascending dose cohorts $(25,75,200 \mathrm{mg} /$ day) were randomized (9:3) to evobrutinib or placebo once daily for 14 days. Safety and tolerability were assessed following single and multiple dosing, and PK parameters determined by non-compartmental methods. Change from baseline in QT interval was obtained from 12 lead electrocardiogram recordings and corrected for heart rate by Fridericias method (QTcF).

Results Treatment-emergent adverse events (TEAEs) with evobrutinib were mostly mild, occurring in $25 \%$ of participants after single dosing, and $48 \%$ after multiple dosing. The nature and incidence of TEAEs were similar among evobrutinib and placebo groups, with no apparent dose relationship regarding the frequency or type of TEAEs. Evobrutinib showed rapid absorption ( $\operatorname{tmax} \sim 0.5$ hour), short half-life ( $\sim 2$ hour), and dose-proportional PK following single and multiple dosing, with no accumulation or time dependency on repeat dosing. ConcentrationQTcF analyses revealed no significant exposureeffect relationship. Based on a linear mixed-effects model for change from baseline in QTcF (QTcF), the slope of the relationship between mean placebo-adjusted QTcF (QTcF) and concentration was negative and close to zero $(0.00027 \mathrm{~ms} / \mathrm{ng} /$ $\mathrm{mL} ; \mathrm{p}=0.86$; figure 1$)$. The predicted $\mathrm{QTcF}$ effect at geometric mean Cmax for the highest dose $(1512 \mathrm{ng} / \mathrm{mL})$ was $1.16 \mathrm{~ms}$, with an upper limit of $3.26 \mathrm{~ms}$ for the $90 \%$ two- 